\title{
Methods of Bargaining in Logistics Processes
}

\author{
Jasmina Pašagić Škrinjar ${ }^{1, *}$, Borna Abramović ${ }^{1}$, and Martina Furdić $^{1}$ \\ ${ }^{1}$ University of Zagreb, Faculty of Transport and Traffic Sciences, Vukelićeva 4, 10000 Zagreb, \\ Croatia
}

\begin{abstract}
This paper outlines the methods of bargaining taken from game theory. The methods and types of bargaining are defined with the aim of establishing a cooperative relationship among the participants to mutual benefit. The methods in the paper are explained under various bargaining conditions and through instances of their application. The paper also examines the application of game theory and methods of bargaining within different logistics processes.
\end{abstract}

\section{Introduction}

Game theory should be understood as means of modelling real-world issues with the aim of ensuring easier decision-making and taking into consideration all actions and their quantification. A model of a game should be encompassing enough so that it can be considered a valid approximation of the actual market situation that is being analysed, but at the same time simple enough so that it is technically suitable for a more specific analysis. There is no algorithm for balancing the two requirements, but a solution is proposed for each specific situation.

Game theory models are based upon the mathematical knowledge and intuition of a researcher about what is relevant for the specific situation, and what is not. A successful application of such models in the analysis of certain logistical and transport issues requires a thorough understanding of the issue being analysed. A concrete analysis demands a multidisciplinary approach, which means it should involve experts from many fields (e.g. mathematicians, logistical process expert, and others). The lack of a team approach might result in deviations in determining optimal strategies.

Game results are commonly expressed in numbers. A larger number indicates that the certain strategy results has a higher score, which in logistics may refer to higher profit, faster delivery, safer transport, a more long-term business, and competitive advantage.

Bargaining games are a separate class of games popular in economic and other analyses, business processes, political action, logistical and transport process, and in daily life.

Person A wants to sell a used forklift for no less than 10,000 €, while Person B wants to purchase such a forklift, ready to pay up to $13,000 €$. If the seller sells the forklift for a price within that range, he makes a profit. If the buyer gets the forklift for the price within his range, he has also benefited. There is, therefore, a space in which both parties are involved in an exchange and thereby ensure mutual benefit.

* Corresponding author: jpasagic@fpz.hr 
Different variables impact the process of negotiation, divided into external and internal. The effect of the external and internal options was defined by Muthoo [1] on the example of marital relationships, but this paper will analyse an example in logistics.

Companies A and B are logistical companies. Company A has a more complete fleet of vehicles and better storages, but a smaller storage capacity than Company B. Furthermore, Company A has a better marketing service and position on the local market. It cooperates with logistical Company B in terms of leaving a part of its business to it and occasionally using its storage facilities (outsourcing). Company B provides services for large international department stores and holds a stronger position on the international market. Both companies take to the external market since it increases their chance of ensuring work and gaining higher profit. In their negotiations, both logistical companies use their respective advantages to achieve a more positive outcome from themselves.

\section{Literature review}

The application of negotiation games has been in used for a number of years in various aspects of business, and the following part will examine the instances of the use of game negotiations in logistics processes. In his work, Reyes [2] applies Shapley's concept on solving the unloading / loading issues in order to maintain stable conditions within the logistics network. Sucky [3] is concerned with supply chain management from the perspective of inventory management. For a buyer to be motivated to order in quantities that are favourable to the supplier, the paper analyses the supplier's ability to introduce a cooperative purchasing policy for the buyer. The research in this paper offers several negotiation models dependent on alternative supplier strategies. With the help of these models, a cooperative relationship between the buyer and supplier can be established to mutual benefit.

The research presented in another paper written by Sucky [4] provides a bargaining model with asymmetric information about the buyers cost structure, assuming that the buyer has the power to impose his individual optimal policy. Plambeck and Taylor $[5,6]$ analyse two similar games between two firms that have an option of pooling their capacity and investments to maximize the total value. In the first stage, firms choose investment into an effort that affects the market size. In the second stage, firms bargain over the division of the market and profits. Esmaeili, Aryanezhad and Zeephongsekul [7] proposed several seller-buyer supply chain models, which incorporate both cost factors, as well as elements of competition and cooperation between seller and buyer. They modelled the relationships between seller and buyer on non-cooperative and cooperative games, and compared the results between different models.

Suh and Park [8] analyse the delay loss cost per ton and per day between the charterer and the ship owner, by determining the equilibrium charterage in bargaining with asymmetric impatience. They used Nash equilibrium theory, which aims at reducing the negotiation time. The analysis made by Wang, Guo and Efstathiou [9] explains noncooperative behaviour in a two-echelon decentralized supply chain, composed of one supplier and $\mathrm{n}$ retailers. They built the approximate decision model of base stock level for sufficient supply from the supplier, in which the suppliers reactions are not considered, and its non-cooperative behaviour is obtained. For insufficient supply from the supplier, a much more complicated non-cooperative behaviour is obtained, and they find that the competition occurred between all the retailers, as well as the supplier. In order to guarantee optimal cooperation in the system, they designed several Nash equilibrium contracts in echelon inventory games and local inventory games. Kwak, Kim and Moon [10] also explored bargaining between suppliers and buyers. They developed two different models: one for the situation where the supplier has a superior bargaining power over the buyer, and the other 
for the reverse situation. For each model, they derived a method to find the best strategy of each agent through analysis based on the game-theoretic approach. The solution they found using that method was verified to be the Nash Equilibrium of each model.

Game theory also finds some interesting problem solution Pašagić Škrinjar, Abramović and Brnjac [11] for planning of urban transport, especially problem of introducing the new bus line. Then Koryagin [12] again use Game theory for urban planning for the Travel Demand Management. Catalano amd Migliore [13] have use game theory for solving the design of logistic terminals. Liu, Wilson and Luo [14] use cooperative game to solve the impact of Panama Canal expansion on the container-shipping market. Babu and Mohan [15] use an integrated approach to evaluating sustainability in supply chains using evolutionary game theory.

\section{Bargaining methods}

The most well-known bargaining solution was put forward by John Nash in 1950 [16]. The author describes the bargaining problem.

Rational individuals $A$ and $B$ willingly enter negotiations. They are familiar with all the outcomes of these negotiations, to which they have attributed various benefits, based on their respective expectations. The negotiations can be concluded in many different ways, and the set of possible agreements represents the permissible area $D$, as seen in Figure 1.

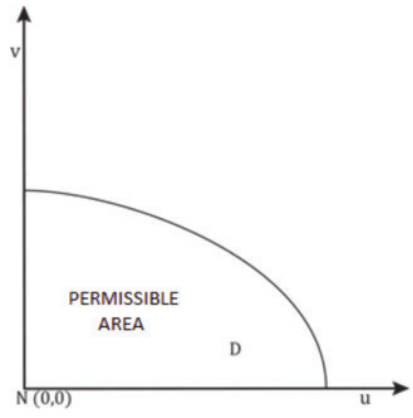

Fig. 1. Permissible area in a bargaining situation. Source: authors

The coordinates $u$ and $v$ in Figure 1 represent the winnings of Player $A$, i.e. Player $B$. The permissible area also contains the failure point (status quo, disagreement point) $N(0,0)$, the negative outcome of bargaining. Disagreement point $N$ is not necessarily located in the origin of the coordinate system. It can be located within the area $D$, denoted by $\left(u_{1}, v_{l}\right)$ in $N\left(u_{l}, v_{l}\right)$. Other points in the permissible area denote the possible agreements which are differently acceptable for both players.

Since the players are perfectly rational, they will not observe all possible outcomes, but rather the ones that are Pareto efficient.

In a Pareto efficient outcome neither individual can make their preference criterion better off without making any other individual's preference criterion worse off. The set of all Pareto efficient allocations (points) comprises negotiating set $P$ within the permissible area, $P \subset D$, as illustrated in Figure 2 .

The allocations of the negotiating set are payoff pairs $(u, v)$, whereby individuals prefer any point in the set $P$. Since both individuals wish to reach an agreement, they are willing to give up on for them most favourable gains in the boundary points of the negotiating set.

A successful conclusion of negotiation refers to only cooperative behaviour that improves the negotiating outcome. 


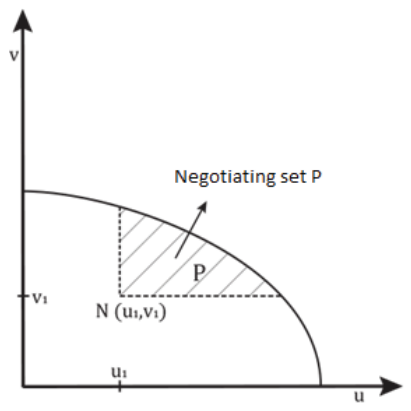

Fig. 2. Negotiating set $P$. Source: authors

In case of an agreement, individuals achieve benefits which are equal $\left(u-u_{l}\right)$ for the first and $\left(v-v_{l}\right)$ the second individual. If the negotiating set contains only one point, the problem becomes trivial and the successful conclusion follows immediately. Therefore, let us assume that the set $P$ contains several points and let us set the problem of allocating the agreement point $\left(u^{*}, v^{*}\right)$.

Nash proposes four axioms which should be satisfied in each negotiating solution:

Pareto-optimum (PO): The accepted allocation must be a unique Pareto optimal solution.

Symmetry $(S)$ : If the permissible area is symmetric, then the allocation of $(a, b)$ includes the allocation of $(b, a)$. If $u_{1}=v_{l}$ for the disagreement point, then the same is true for the agreement point, $u^{*}=v^{*}$.

Invariant to equivalent utility representations $(E P)$ : Assume that the payoff functions of the two individuals, $u(\cdot)$ and $v(\cdot)$, based on which the agreement point was allocated: $\left(u^{*}\right.$, $v^{*}$ ). If we apply positive linear transformation and transform their payoff functions into new functions:

$u(\cdot)=a_{1} u(\cdot)+b_{1}$, where $a_{1}$ and $b_{1}$ are positive constants,

$v^{\prime}(\cdot)=a_{2} v(\cdot)+b_{2}$, where $a_{2}$ and $b_{2}$ are positive constants,

then the new agreement point represents the linear transformation of the preceding point, $\left(a_{1} u^{*}+b_{1}, a_{2} v^{*}+b_{2}\right)$. This agreement point remains the same, now expressed in payoffs measured by the new scales.

Independence of irrelevant alternatives (IA): Let two problems in negotiating have the same conflict points and let that negotiating set of the 2 is within the negotiating set of 1 . If the agreement point of $1\left(u_{1}^{*}, v_{1}{ }^{*}\right)$ is located within the negotiation set of 2 , then it is at the same time the agreement point for $2\left(u_{1}^{*}, v_{1}^{*}\right)=\left(u_{2}^{*}, v_{2}^{*}\right)$.

Based on these four conditions, Nash proved the following theorem:

If conditions $P O, S, E P$, and $I A$ have been met, then the agreement point $\left(u^{*}, v^{*}\right)$ is the solution of the problem:

$$
\max \left(u-u_{1}\right)\left(v-v_{l}\right)
$$

in the negotiating set, if there are points that prove $u>u_{l}$ and $v>v_{l}$.

The obtained solution is referred to as Nash's bargaining solution and is the only one that satisfies all the four axioms.

Nash's bargaining solution can be applied in various forms of bargaining, some of which include symmetric games, asymmetric bargaining games, different scales for determining payoffs, various risk forms in bargaining, and many others. 


\subsection{Example 1: Asymmetric bargaining games}

The first asymmetry concerns the starting positions of the players. Assume that the disagreement point $N$ is not $(0,0)$ and that Player $A$ or Player $B$ can count on a definite profit on the side, in case the negotiation fails. If Player $A$ has a guaranteed amount of $250 €$, and the profit of Player $B$ depends exclusively on the outcome of the observed negotiation, the disagreement point $N$ is $(250,0)$ and it affects the final outcome. It cannot be expected that the players divide the money into equal parts, as seen in Figure 3.

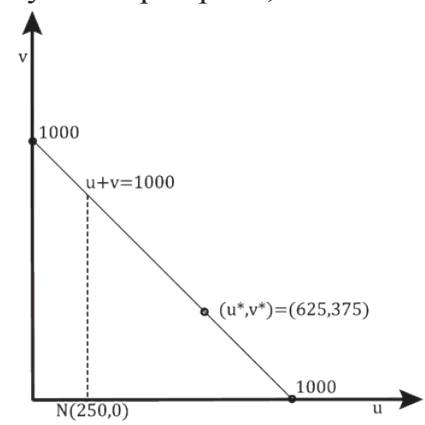

Fig. 3. Asymmetric bargaining games. Source: authors

Assuming that players are still neutral towards risk, which makes their payoffs equal to financial outcomes $u=x, v=y$, therefore the efficient distribution is $u+v=x+y=1000$. The bargaining solution is the solution of the problem:

$$
\begin{gathered}
\max \left(x-n_{1}\right)\left(y-n_{2}\right) \\
\text { that is, } \\
\max (x-250)(y-0)=\max (x y-250 y) \\
\text { for } y=1000-x
\end{gathered}
$$

in the expression above:

$$
\max (1250 x-x 2-250000)
$$

A zero first derivative of $1250-2 x=0$, is $x=625$, and $y=375$. The result $(625,375)$ shows that the concluded agreement is asymmetrical in favour of the player that enters bargaining with a more favourable starting position [17].

\subsection{Example 2: Negotiator's attitude towards risk}

So far, it has been assumed that negotiators are not prone to taking risk, which meant that in the case of symmetric disagreement points $N=(0,0)$ the available sum was divided into equal parts. The negotiator's attitude towards risk affects the bargaining outcome, because it shapes the payoff functions of the negotiator. Let us assume that Player $A$ is still neutral towards risk, and let $u=x$, while Player $B$ shows a slight animosity towards risk, so that the sums y are added payoffs which are $v=y^{4 / 5}$, i.e. $y=v^{5 / 4}$. By substituting these payoffs for lines $x+y=1000$, there follows a curve $u+v^{5 / 4}=1000$, as seen in Figure 4. 


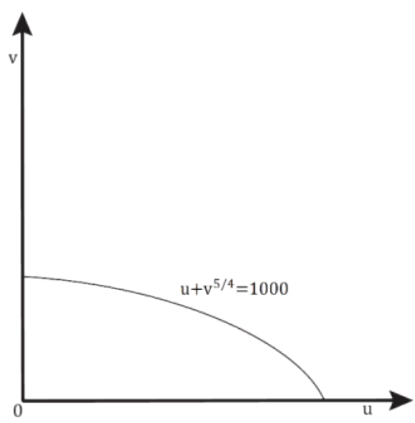

Fig. 4. Negotiator's attitude towards risk. Source: authors

Substituting $v=(1000-u)^{4 / 5}$ into $\max \left(u-n_{1}\right)\left(v-n_{2}\right)$ it follows: $\max u(1000-u)^{4 / 5}$ let first derivative be 0 :

$$
\begin{gathered}
(1000-u)^{4 / 5}+(4 / 5) u(1000-u)^{4 / 5-1}(-1)=0 \\
(1000-u)^{4 / 5}=(4 / 5) u(1000-u)^{-1 / 5}
\end{gathered}
$$

and multiplying the first and the second equality by expression $(1000-u)^{1 / 5}$, it follows:

$$
1000-u=(4 / 5) u
$$

after the calculation we determine the bargaining result for an individual who is neutral towards risk, $u=x=555.5$, which is followed immediately by $y=1000-555.5=444.5 €$.

The solution of the bargaining game $(555.5 ; 444.5)$ shows that the different attitude of the negotiator towards risk conditions unequal distribution. When the negotiator shows animosity towards risk, the fear of failure makes him bargain unfavourably away from equal distribution of income. The higher his animosity towards risk, the more unfavourable the distribution of income. If Player $A$ is neutral, and $u=x$, and Player $B$ shows animosity towards risk so that his payoff function equals $v=y^{1 / 2}$, the solution of the bargaining is (666.6; 333.3).

The inequalities of distribution are even more evident if Player $A$ is prone to risk, and Player $B$ against it. The final outcome is then substantially asymmetrical in favour of Player $A[17]$.

Although logical, such result evokes much criticism, even contestation of Nash's solution. These solutions favour individuals prone to risk, which is why certain authors state that it encourages players to falsely demonstrate individual preferences. It is in the interest of rational individuals who are neutral or show animosity towards risk to conceal their preferences and enter negotiations as players prone to risk. Nash's solution only makes sense if there's an assumption that it is based on real and not false payoff functions of both negotiators.

The initial assumption about perfectly rational and intellectually equal partners is what separates game theory from practice. The theory completely disregards the individuality of players, even though knowledge, intelligence and character traits affect the final outcome of a negotiation in real-world situations. The theoretical solution is based solely on the specificity of conflict situation.

\subsection{Example of negotiating in channels of distribution}

This example is based on building the basic bargaining model in which a manufacturer (with utility $u_{M}$ ) sells to a retailer (who is similar to a news vendor with utility $u_{R}$ ). The players negotiate on the terms of trade, and effectively the utility allocations. The retailer faces a random demand, the density of which is $f(\varepsilon)$. He has to place an order of $Q$ units in 
anticipation of the demand. He incurs purchasing, holding, and shortage costs, and gains revenue proportional to the quantity he sells. We will denote his revenue per unit as $s$, the other costs per unit as $w, h$ and $v$, respectively. The manufacturer faces a production cost per unit, denoted as $c$, and sells to the retailer at a wholesale price $w$. The profit, given a demand realization $\varepsilon$, of the retailer and the manufacturer as $\pi_{R}(w, Q, \varepsilon)$ and $\pi_{M}(w, Q, \varepsilon)$, respectively. With a slight abuse of notation, we denote the expected profits as $E\left[\pi_{R}(w, Q, \varepsilon)\right]=\pi_{R}$ and $E\left[\pi_{M}(w, Q, \varepsilon)\right]=\pi_{M}$, by simply dropping $\varepsilon$ from the arguments. For the sake of exposition, we describe the construction of the game using wholesale price contracts. Recall that a NB game requires us to identify a feasible set of payoffs and a disagreement point that are pre-determined and are independent of the negotiations. To do so, let us first assume that the supplier and the retailer negotiate on $(w, Q)$. The feasible set is constructed as follows: we first define:

$$
\Delta=\left\{\left(E\left(u_{M}\left(\pi_{M}(w, Q, \varepsilon)\right)\right), E\left(u_{R}\left(\pi_{R}(w, Q, \varepsilon)\right)\right)\right):(w, Q) \in T\right\}
$$

for some suitable compact set $T \subseteq R$. The set $T$ is assumed to be a rectangular set. This is be the case, for instance, when both the wholesale price and the purchasing quantity are allowed to take values in a closed interval in the positive real axis. We let $\Omega$ be the convex hull of $\Delta$ and we henceforth refer to $\Omega$ as the feasible set.

The disagreement outcomes have several interpretations, depending on the context. They represent a certain level of expected utility that each player requires as a minimum level of acceptance to participate in the trade. We set this to zero. In a setting where players are risk neutral, this is without loss of generality, i.e. the structure of the results trivially change. When players are risk averse, they need to be a bit more careful. However, the results for the system above (a manufacturer - retailer chain) in this paper essentially remain the same.

When players are risk-neutral and negotiate on both $w$ and $Q$, a few observations can be made. First, the bargaining solution of the game will not be a randomized outcome. This is because risk neutrality implies $\Delta=\Omega$. Furthermore, note that the bargaining solution necessarily requires Pareto optimality as a condition. This immediately ensures that when the players are risk-neutral, the negotiated quantity is always the coordinating quantity, $Q_{c}$. This simplifies the analysis and removes double marginalization effects. The negotiated value of $w$ allocates the channel surplus between the two players. This simple observation continues to hold when more complex contracts are considered (i.e., buyback, revenue sharing, etc.), as long as one assumes that all parameters of the contract are simultaneously negotiated [18].

\section{Conclusion}

Game theory does not adopt the role of an advisor, nor does it give directions to players on how to win. The main goal of the theory is to identify and model the essence of conflict which is specific in a certain situation. The initial assumption about perfectly rational and intellectually equal players is what separates theory from practice. The former completely disregards individual impacts of players, whilst in real-world negotiating circumstances the knowledge, intelligence and character traits of the negotiator indeed determine the final outcomes. The theoretical solution, i.e. solution proposal is determined solely on the specificity of the conflicting situation.

Since theory observes only the perfectly rational individuals that always select from a set of optimal solutions, the Pareto-optimum is accepted theoretically. In real-world negotiation circumstances, the rationality condition is infringed, so final outcomes of numerous negotiations do not represent the Pareto efficient solutions. Decision-makers often disregard the assumptions of a perfectly rational decision-making, while the 
complexity of negotiating situations makes it impossible to precisely determine the Pareto optimal solutions set. The symmetricity condition in a bargaining game should be ensured so that negotiation is a fair game. If the players are in opposite and completely symmetrical positions, then a deal is fair when both obtain equal benefits. The condition understands symmetricity of results expressed in benefits, which does not implicitly state that they are equal in monetary or some other expression.

The paper is supported by the PROM - PRO research project "Adapting the railway system in the integrated passenger transport (ARSIPT)", that is solved at Faculty of Transport and Traffic Sciences, University of Zagreb.

This paper is supported by the research project "From horse-drawn railway to intermodal transport" within Visegrad Fund.

\section{References}

1. A. Muthoo, World Economics 1, 2, 145-166 (2010)

2. P. Reyes, Applied Mathematics and Computation 168, 2, 1419-1431 (2005), DOI: 10.1016/j.amc.2004.10.030

3. E. Sucky, International Journal of Production Economics 93-94, 253-262 (2005), DOI: 10.1016/j.ijpe.2004.06.025

4. E. Sucky, European Journal of Operational Research 171, 2, 516-535 (2006), DOI: 10.1016/j.ejor.2004.08.039

5. E.L. Plambeck, T.A. Taylor, Management Science 53, 12, (2001)

6. E.L. Plambeck, T.A. Taylor, Management Science 51, 1, (2005)

7. M. Esmaeili, M.B. Aryanezhad, P. Zeephongsekul, European Journal of Operational Research 195, 2, 442-448 (2009), DOI: 10.1016/j.ejor.2008.02.026

8. S. Suh, N. Park, The Asian Journal of Shipping and Logistics 26, 2, 247-261 (2010), DOI: 10.1016/S2092-5212(10)80004-5

9. H. Wang, M. Guo, J. Efstathiou, European Journal of Operational Research 157, 2, 372-388 (2004), DOI: 10.1016/S0377-2217(03)00233-9

10. T.C. Kwak, J.S. Kim, C. Moon, Computers \& Industrial Engineering 51, 2, 219-228 (2006), DOI: 10.1016/j.cie.2006.02.004

11. J. Pašagić Škrinjar, B. Abramović, N. Brnjac, Tehnicki Vjesnik - Technical Gazette 22, 6, 1617-1621 (2015)

12. M. Koryagin, Periodica Polytechnica Transportation Engineering, (to be publish)

13. M. Catalano, M. Migliore, Journal of Transport Geography 41, 63-73 (2014), DOI: 10.1016/j.jtrangeo.2014.08.010

14. Q. Liu, W.W. Wilson, M. Luo, Maritime Policy \& Management 43, 2 (2016), 209-221, DOI: $10.1080 / 03088839.2015 .1131863$

15. S. Babu, U. Mohan, Computers \& Operations Research 89, 269-283 (2018), DOI: 10.1016/j.cor.2017.01.008

16. J.F. Nash, Jr., Econometrica 18, 2, 155-162 (1950)

17. D. Pavličić, Teorija odlučivanja (Ekonomski fakultet, Belgrade, 2010)

18. M. Nagarajan, G. Sosic, European Journal of Operational Research 187, 3, 719-745 (2008), DOI: 10.1016/j.ejor.2006.05.045 